\title{
THEORY OF PENGUINS IN B DECAYS
}

\author{
N.G. Deshpande \\ Institute of Theoretical Science \\ University of Oregon \\ Eugene, OR 97403
}

The theory of loop induced rare B decays is reviewed. Both electromagnetic penguin processes and gluon mediated penguin processes are discussed. After considering $b \rightarrow s \gamma$ and $b \rightarrow s e^{+} e^{-}$decays, purely hadronic modes like $B \rightarrow K \phi$ are estimated. CP violation in charged B Decays is reviewed. The asymmetries for pure penguin processes are estimated first. A larger asymmetry can result in those modes where a tree diagram and a penguin diagram interfere, however these estimates are necessarily model dependent.

Rare decays of the B meson offer a unique opportunity to study electroweak theory in higher orders. Process like $b \rightarrow s \gamma, b \rightarrow s e^{+} e^{-}$and $b \rightarrow s g$ do not occur at the tree level, and at one loop level they occur at a rate small enough to be sensitive to physics beyond the standard model. Similar flavor violating processes in the $\mathrm{K}$ system have the disadvantage that long distance effects are quite large, and it is difficult to extract the quark level physics from the well known process [1] like $K^{+} \rightarrow \pi^{+} e^{+} e^{-}$and $K_{L} \rightarrow \pi^{\circ} e^{+} e^{-}$. However, although the quark level calculations are fairly precise in the $b$ quark system, one is still hampered by the lack of knowledge of hadronic form factors, and by a reliable technique of evaluating interesting modes from four quark operators. We shall use some of the wave function results based on reference $[2,3]$. In recent years there has been an improvement in form factor evaluation using heavy quark techniques [4] and from improving lattice model calculations.

On the experimental side, recent observation [5] by CLEO of the exclusive decays of $B \rightarrow K^{*} \gamma$,

$$
B R\left[B \rightarrow K^{*} \gamma\right]=(4.5 \pm 1.5 \pm 0.9) \times 10^{-5}
$$

has provided the first evidence of the electromagnetic penguin process. This exciting discovery has led to much theoretical activity, and further penguin processes are eagerly anticipated.

\section{Electroweak Penguin Processes}

\subsection{The process $b \rightarrow s \gamma$}

The process $b \rightarrow s \gamma$ proceeds with $\mathrm{W}$ boson and $u, c, t$ quarks in the loop as shown in Fig. 1. The purely electromagnetic one loop result can be obtained from [6]. The two loop correction coming from QCD however enhances the rate significantly

Contribution to second edition of "B Decays" ed. S. Stone; publisher, World Scientific. 

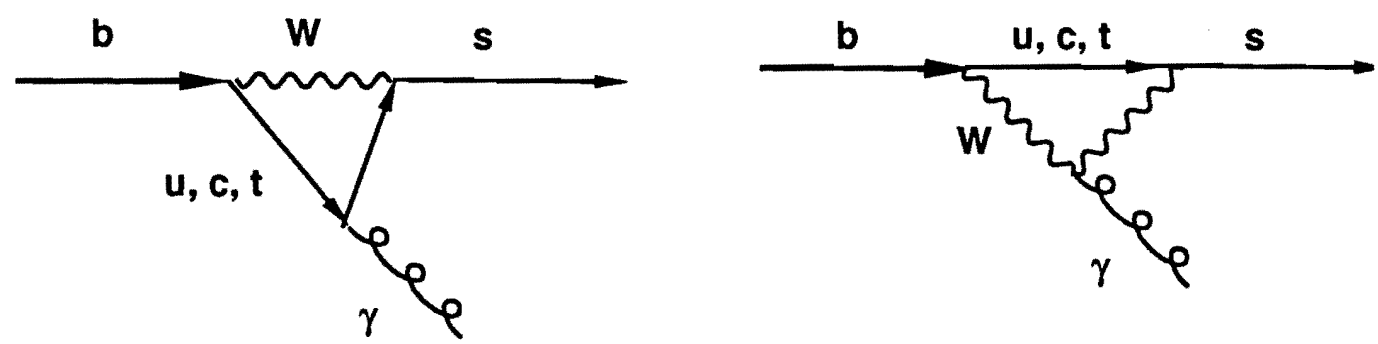

Figure 1: Diagrams for the process $b \rightarrow s \gamma$

as was first noted in Ref. [7,8]. The QCD corrections for light quarks were carried out in Ref. [9], and for heavy quarks were done in Ref. [10,11]. The effective Hamiltonian for this process in the notation of $[10]$ is

$$
H_{e f f}=\frac{4 G_{F}}{\sqrt{2}}\left(V_{b t} V_{s t}^{*}\right) c_{7}\left(m_{b}\right) O_{7}
$$

where

$$
O_{7}=\left(e / 16 \pi^{2}\right) m_{b} \bar{s}_{L} \sigma_{\mu \nu} b_{R} F^{\mu \nu}
$$

and

$$
\begin{aligned}
c_{7}(m) & =\rho^{-16 / 23}\left[c_{7}\left(m_{W}\right)-\frac{8}{3} c_{8}\left(m_{W}\right)\left(1-\rho^{2 / 23}\right)+\frac{232}{513}\left(1-\rho^{19 / 23}\right)\right], \\
c_{7}\left(m_{W}\right) & =(-1 / 2)\left(x / z^{3}\right)\left[2 x^{2} / 3+5 x / 12-7 / 12-\left(\left(3 x^{2} / 2-x\right) / z\right) \ln x\right] \\
c_{8}\left(m_{W}\right) & =(-1 / 2)\left(x / 2 z^{3}\right)\left[x^{2} / 2-5 x / 2-1+(3 x / z) \ln x\right]
\end{aligned}
$$

where $m=m_{b}, x=\left(m_{t} / m_{W}\right)^{2}, z=x-1$ and $\rho=\alpha_{s}(m) / \alpha_{s}\left(M_{W}\right)$

The rate for $b \rightarrow s \gamma$ neglecting s quark mass is given by

$$
\Gamma(b \rightarrow s \gamma)=\frac{G_{F}^{2} \alpha m_{b}^{5}}{32 \pi^{4}}\left|c_{7}\right|^{2}\left|V_{b t} V_{s t}^{*}\right|^{2}
$$

When comparing with experiment, it is much better to compute the ratio

$$
\frac{\Gamma(b \rightarrow s \gamma)}{\Gamma(b \rightarrow c e \nu)}=\frac{6 \alpha}{\pi \eta \lambda}\left|c_{7}\right|^{2}
$$

Where $\eta=1-8 r^{2}+8 r^{6}-r^{8}-24 r^{4} \ell n r$ and $\lambda=1-2 \alpha_{s}\left(m_{b}\right)(2.4) / \pi$ and $r=m_{c} / m_{b}$. The semileptonic $B R(b \rightarrow c e \nu) \approx 0.107$.

Recently Buras et. al. [12] have discussed the theoretical uncertainities in the above formula. They estimate the uncertainities from the choice of QCD scale (taken above as $m_{b}$ ) to be significant, and can be as large as $\pm 25 \%$. To reduce these theoretical uncertinities a three loop calculation is necessary. We reproduce their 
estiamte of $B R(b \rightarrow s \gamma)$ as a function of $m_{t}$ with their estimate of uncertainities in Fig. 2. The present inclusive $B R\left(B \rightarrow X_{s} \gamma\right)$ from CLEO:

$$
B R\left[B \rightarrow X_{s} \gamma\right]<5.4 \times 10^{-4}(95 \% \text { C.L. })
$$

is also shown in the figure.

The exclusive process $B \rightarrow K^{*} \gamma$ provides a clean signature for the process $b \rightarrow s \gamma$. The long distance contribution is expected to be small for this process. We need the following hadronic matrix elements, where we have written only those terms that are allowed in the matrix element.

$$
\begin{gathered}
\left\langle K^{*}(k)\left|\bar{s} \sigma_{\mu \nu} q^{\nu} \frac{\left(1+\gamma_{5}\right)}{2} b\right| B(p)\right\rangle=i \epsilon_{\mu \nu \lambda \sigma} \epsilon^{\nu}(k)(p+k)^{\lambda}(p-k)^{\sigma} T_{1}\left(q^{2}\right) \\
+\left[\epsilon_{\mu}(k)\left(m_{B}^{2}-m_{K^{*}}^{2}\right)-(\epsilon \cdot q)(p+k)_{\mu}\right] T_{2}\left(q^{2}\right)
\end{gathered}
$$

The values of $T_{i}$ at $q^{2}=0$ are far away from the zero recoil point $q^{2}=\left(m_{B}-m_{K^{*}}\right)^{2}$. A conservative extrapolation [13] yields

$$
T_{1}(0)=T_{2}(0)=0.115
$$

The ratio of the exclusive to incluse process gives

$$
\frac{\Gamma\left(B \rightarrow K^{*} \gamma\right)}{(b \rightarrow s \gamma)}=2\left(\frac{m_{B}}{m_{b}}\right)^{3}\left(1-\frac{m_{K^{*}}^{2}}{m_{B}^{2}}\right)\left\{T_{1}^{2}+T_{2}^{2}\right\} \approx .06
$$

Other older estimates range from $30 \%$ to $4 \%[14]$. Recently, new estimates for this ratio have been obtained. Using effective lagrangian for heavy and light mesons, Casalbuoni et al. [15] obtain form factors in close agreement with our values quoted above. A comprehensive study by Ali et. al. [16] find a range of $3.5 \%$ to $12.2 \%$. A lattice gauge calculation of Bernard et. al. [17] find for $R_{K^{*}}$ the ratio of exclusive to inclusive process:

$$
R_{K^{*}}=(6.0 \pm 1.2 \pm 3.4) \%
$$

The experimental value in Equation (1) is consistent with these estimates within quoted errors.

The process $b \rightarrow s \gamma$ has been used to put limits on contributions arising from physics beyond the standard model. Contributions from fourth generation [18], from susy paticles [19], from two Higgs doublet models [20] and anomalous gauge couplings[21] have all been considered. As the inclusive process is measured and errors are reduced, we anticipate these limits becoming more stringent.

\subsection{The Processes $b \rightarrow s \ell^{+} \ell^{-}$}

This process occurs through $W$ boson box diagram, $Z$ exchange, and photon exchange. The effective Hamiltonian density relevant for $b \rightarrow s l^{+} l^{-}$decay is:

$$
H_{\text {eff }} \cong \frac{4 G_{F}}{\sqrt{2}}\left(V_{b c} V_{c s}^{*}\right) \sum_{j=1,2,7, \cdots, 10} c_{j}(m) O_{j}(m)
$$




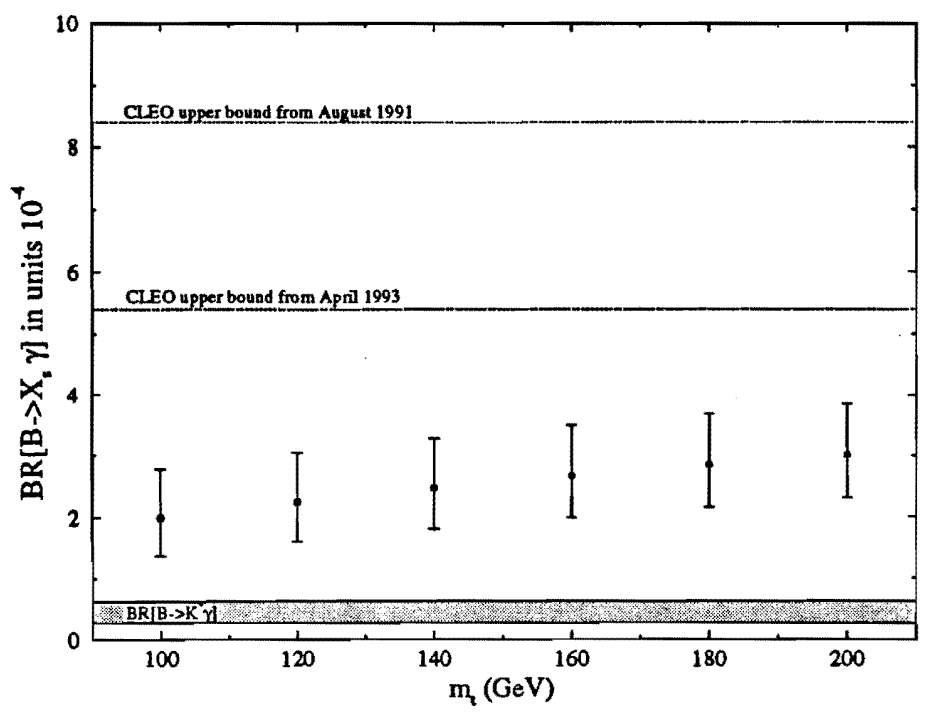

Figure 2: BR for $b \rightarrow s \gamma$ as functions of $m_{t}$ with theoretical uncertainities from $\operatorname{Ref}[12]$

The important operators for us are:

$$
\begin{aligned}
O_{1} & =\bar{s}_{L}^{i} \gamma_{\mu} b_{L}^{j} \bar{c}_{L}^{j} \gamma^{\mu} c_{L}^{i}, \\
O_{2} & =\bar{s}_{L}^{i} \gamma_{\mu} b_{L}^{i} \bar{c}_{L}^{j} \gamma^{\mu} c_{L}^{j}, \\
O_{7} & =\left(e / 16 \pi^{2}\right) m_{b}\left(\bar{s}_{L}^{i} \sigma_{\mu \nu} b_{R}^{i}\right) F^{\mu \nu}, \\
O_{8} & =\left(g / 16 \pi^{2}\right) m_{b}\left(\bar{s}_{L}^{i} \sigma_{\mu \nu} T_{a}^{i j} b_{R}^{j}\right) G_{a}^{\mu \nu}, \\
O_{9} & =\left(e^{2} / 16 \pi^{2}\right)\left(\bar{s}_{L}^{i} \gamma_{\mu} b_{L}^{i}\right) \bar{\ell} \gamma^{\mu} \ell, \\
O_{10} & =\left(e^{2} / 16 \pi^{2}\right)\left(\bar{s}_{L}^{i} \gamma_{\mu} b_{L}^{i}\right) \bar{\ell} \gamma^{\mu} \gamma_{5} \ell .
\end{aligned}
$$

Here $F_{\mu \nu}$ and $G_{\mu \nu}^{a}$ are the electromagnetic and gluon interaction field strength tensors, respectively, and $e$ and $g$ are the corresponding coupling constants.

The QCD-renomalized coefficients $c_{j}(m)$ calculated in Ref.'s [22,10] are:

$$
\begin{aligned}
c_{1}(m)= & (-1 / 2)\left(\rho^{-6 / 23}+\rho^{12 / 23}\right), \\
c_{2}(m)= & (-1 / 2)\left(\rho^{-6 / 23}-\rho^{12 / 23}\right), \\
c_{7}(m)= & \rho^{-16 / 23}\left[c_{7}\left(m_{W}\right)-\frac{8}{3} c_{8}\left(m_{W}\right)\left(1-\rho^{2 / 23}\right)+\frac{232}{513}\left(1-\rho^{19 / 23}\right)\right], \\
c_{9}(m)= & c_{9}\left(m_{W}\right)-\left[c_{1}(m)+3 c_{2}(m)\right] \ln \left[\left(m_{c} / m\right)^{-8 / 9}\right]- \\
& -\frac{4 \pi}{\alpha_{s}\left(m_{W}\right)}\left[\frac{4}{33}\left(1-\rho^{-11 / 23}\right)-\frac{8}{87}\left(1-\rho^{-29 / 23}\right)\right], \\
c_{10}(m)= & c_{10}\left(m_{W}\right)
\end{aligned}
$$


where as before $m=m_{b}$ and $\rho=\alpha_{s}(m) / \alpha_{s}\left(m_{W}\right)$.

The coefficients at the scale $m_{W}$ receive contribution from $W$ loops. These are:

$$
\begin{aligned}
c_{7}\left(m_{W}\right) & =(-1 / 2) A(x) \\
c_{8}\left(m_{W}\right) & =(-1 / 2) E(x), \\
c_{9}\left(m_{W}\right) & =\left(-1 / s_{W}^{2}\right)[B(x)-C(x)]-4 C(x)-D(x)+4 / 9 \\
c_{10}\left(m_{W}\right) & =\left(1 / s_{W}^{2}\right)[B(x)-C(x)] .
\end{aligned}
$$

The functions $A, \ldots, E$ are:

$$
\begin{aligned}
A(x)= & \left(x / z^{3}\right)\left[2 x^{2} / 3+5 x / 12-7 / 12-\left(\left(3 x^{2} / 2-x\right) / z\right) \ln x\right] \\
B(x)= & (x / 4 z)[-1+(\ln x) / z] \\
C(x)= & (x / 4 z)[x / 2-3+((3 x / 2+1) / z) \ln x] \\
D(x)= & \left(1 / z^{3}\right)\left[-19 x^{3} / 36+25 x^{2} / 36\right. \\
& \left.+\left(\left(-x^{4} / 6+5 x^{3} / 3-3 x^{2}+16 x / 9-4 / 9\right) / z\right) \ln x\right] \\
E(x)= & \left(x / 2 z^{3}\right)\left[x^{2} / 2-5 x / 2-1+(3 x / z) \ln x\right]
\end{aligned}
$$

where $x=\left(m_{t} / m_{W}\right)^{2}, z=x-1$.

The branching ratio of $b \rightarrow s l^{+} l^{-}$can be written after normalizing the rate to $B R(b \rightarrow c e \bar{\nu}) \approx 0.107,[24,22]:$

$$
B R\left(b \rightarrow s l^{+} l^{-}\right)=K\left[F_{1}\left(\left|c_{9}\right|^{2}+\left|c_{10}\right|^{2}\right)+F_{3} c_{9} c_{7}+F_{2}\left|c_{7}\right|^{2}\right],
$$

where

$$
K=(\alpha / 4 \pi)^{2}(2 / \lambda \eta) B R(b \rightarrow c e \bar{\nu})=1.6 \cdot 10^{-7}
$$

and $\alpha$ is fine structure constant. The phase space factor $\eta$ and the $Q C D$ correction factor $\lambda$ for the semileptonic process are as in prvious section. We have used $\eta=$ 0.507 and $\lambda=0.888$. The phase space integration from $\min =\left(2 m_{\ell} / m_{b}\right)^{2}$ to max $=\left(1-m_{s} / m_{b}\right)^{2}$ give the following values [23] for the constants $F_{i}$ :

$$
\begin{gathered}
F_{1}=1, \quad F_{3}=8, \quad \text { for } \quad \text { min } \cong 0, \quad \text { max } \cong 1, \\
F_{2}=32\left[\ln \left(m_{b} / 2 m_{\ell}\right)\right] ; \text { for } \max \cong 1 ; \quad \ell=e, \mu .
\end{gathered}
$$

We plot in Fig. 3 the branching ratios for $b \rightarrow s l^{+} l^{-}$as a function of the top mass for the standard model [24]. In the SM the process $b \rightarrow s e^{+} e^{-}$is enhanced over $b \rightarrow s \mu^{+} \mu^{-}$by $\sim 60 \%$ for $m_{t}=150 \mathrm{GeV}$ due to the small electron mass [24]. Note also that exclusive modes $B \rightarrow \mathrm{Kl}^{+} l^{-}$and $B \rightarrow K^{*} l^{+} l^{-}$would be smaller in the standard model by $8 \%$ and $20 \%$ of the $b \rightarrow s l^{+} l^{-}$respectively, due to the form factor supression [24]. We see that the present bound [25] $B R\left(b \rightarrow s \mu^{+} \mu^{-}\right)<5 \cdot 10^{-5}$ leads to the bound on top of $\sim 390 \mathrm{GeV}$. The curve also shows the dramatic rise in the branching ratio as a function of top mass, and illustrates how an improvement of the experimental bound can significantly improve the top bound. The effect of resonances on $b \rightarrow s l^{+} l^{-}$has also been considered in [23] 


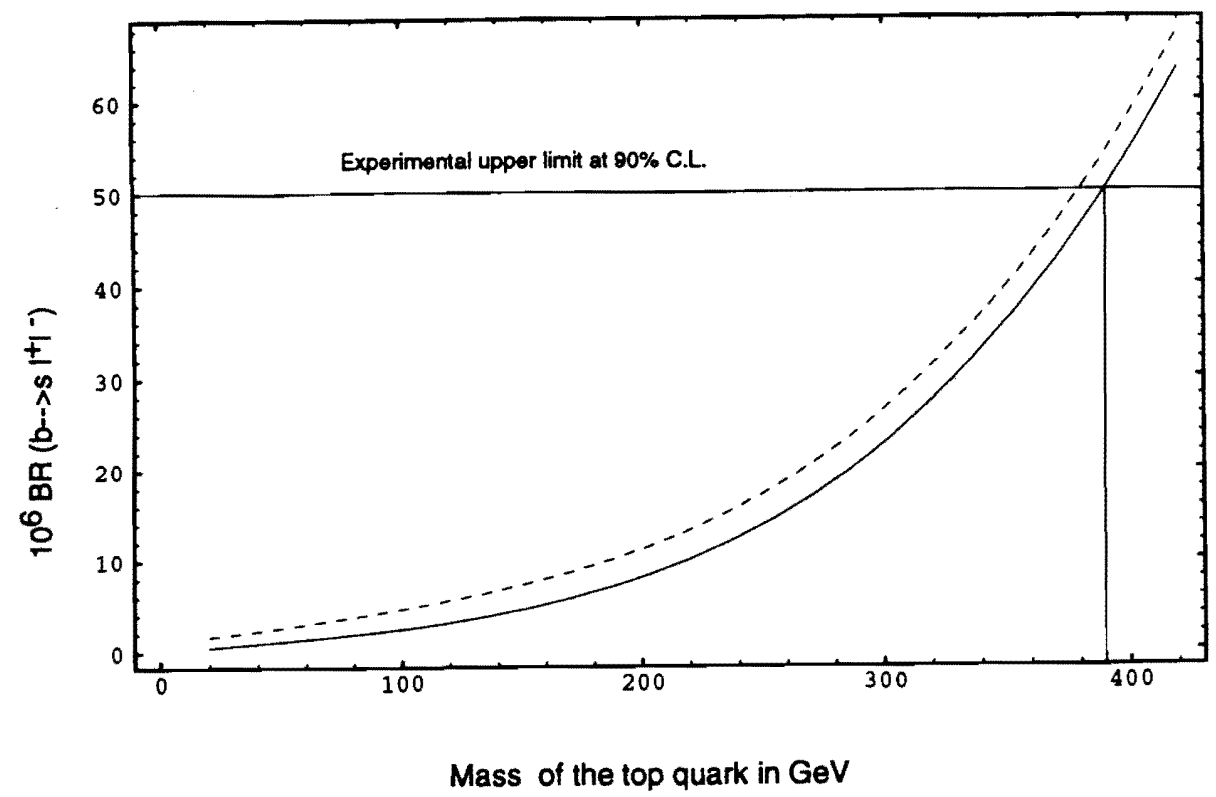

Figure 3: Branching ratios for $b \rightarrow s e^{+} e^{-}$(dashed line) and $b \rightarrow s \mu^{+} \mu^{-}$(solid line) as a function of the top mass. Present bound of $5 \cdot 10^{-5}$ for $b \rightarrow s \mu^{+} \mu^{-}$yields a limit of $390 \mathrm{GeV}$ for $m_{t}$.

\section{Gluon Mediated Penguin Processes}

The process at the quark level involving gluons, $b \rightarrow s g$ and $b \rightarrow s q \bar{q}$ have been considered in Ref $[26,27]$. However, the experimental signature for such a transition is a charmless exclusive mode like $B \rightarrow K \pi$, etc. The estimates for these processes involve matrix elements of four quark operators, and there are difficult to estimate. An added complication here is that charmless hadronic decays can also arise through the tree Hamiltonian with $b \rightarrow u$ transition. A careful study of the modes reveals some where penguin clearly dominates, while others where tree contribution can be significant.

The calculation proceeds in two steps[28]. First we obtain the effective short distance interaction including one-loop gluon mediated diagram. We then use the factorization approximation to derive the hadronic matrix elements by saturating with vacuum state in all possible ways. The resulting matrix elements involve quark bilinears between one meson and vacuum and between two meson states. These are estimated using relativistic quark model wave functions. Such a technique has been used extensively by Ref. [2] for $B$ and $D$ nonleptonic decays and results are in fair agreement with experiment.

We shall first discuss the gluon mediated penguin contribution. Dictated by gauge invariance, the effective flavor changing neutral current $J \mu$ contains two terms. First, proportional to $G_{1}$, we call the charge radius, while the second one, 
proportional to $G_{2}$, is called dipole moment operator.

$$
J_{\mu}=\left(\frac{g_{s}}{4 \pi^{2}}\right)\left(\frac{G_{F}}{\sqrt{2}}\right) \bar{s}^{-i} \frac{1}{2} \lambda_{i j}\left[G_{1}\left(q^{2} \gamma_{\mu}-q_{\mu} q \cdot \gamma\right) L+i G_{2} \omega_{\mu \nu} q^{\nu}\left(m_{s} L+m_{b} R\right)\right] b^{j}
$$

Here $G_{1,2}$ are functions of $K-M$ angles and quark masses:

$$
\begin{aligned}
& G_{1}=\sum V_{k} G_{1}^{k}\left(x_{k}\right), V_{k}=U_{k b} U_{k s}^{*}, k=u, c, t \\
& G_{1}^{k}=\left(\frac{x_{k}}{12}\right)\left(\frac{1}{y_{k}}+\frac{13}{y_{k}^{2}}-\frac{6}{y_{k}^{3}}\right)+\left[\frac{2}{3 y_{k}}-\left(\frac{x_{k}}{6}\right)\left(\frac{4}{y_{k}^{2}}+\frac{4}{y_{k}^{3}}-\frac{3}{y_{k}^{4}}\right)\right] \ln x_{k},
\end{aligned}
$$

where $x_{k}=m_{k}^{2} / m_{w}^{2}$ and $y_{k}=1-x_{k}$.

Note that when the gluon is on-shell, (i.e. $q^{2}=0$ ) the $G_{1}$ contribution vanishes. In the $q^{2} \neq 0$ cases both terms participate. For a gluon exchange diagram (i.e. for the process $b \rightarrow s \bar{q} q$ ) we find $G_{1}$ contribution dominates over $G_{2}$, and we can neglect $G_{2}$. We also evaluate $G_{1}$ at $q^{2}=0$, which is a good approximation. At larger $q^{2}, G_{1}$ develops a small imaginary part, which is important for discussion of CP violation. We shall discuss $\mathrm{CP}$ violation later.

From one-gluon exchange diagram we find the following effective Hamiltonian (local 4-quark operator):

$$
\begin{aligned}
H_{e f f}^{P}= & \kappa\left[\bar{s}_{L}^{i} \gamma_{\mu} b_{L}^{i} \bar{q}_{L}^{j} \gamma_{\mu} q_{L}^{i}-3 \bar{s}_{L}^{i} \gamma_{\mu} b_{L}^{j} \bar{q}_{L}^{j} \gamma_{\mu} q_{L}^{i}\right. \\
& \left.+\bar{s}_{l}^{i} \gamma_{\mu} b_{L}^{i} \bar{q}_{R}^{j} \gamma_{\mu} q_{R}^{j}-3 \bar{s}_{l}^{i} \gamma_{\mu} b_{L}^{j} \bar{q}_{R}^{j} \gamma_{\mu} q_{R}^{i}\right]
\end{aligned}
$$

where

$$
\kappa=\left(\alpha_{s} / 6 \pi\right)\left(G_{F} / \sqrt{2}\right) G_{1}, \alpha_{s}=g_{s}^{2} / 4 \pi
$$

Here $q$ runs over all quark species, although only $u, d$ and $s$ are relevant for our discussion. Charmless decays can also arise from the standard tree level interactions with $b \rightarrow u$ transition. This is given by

$$
\begin{gathered}
H_{e f f}^{T}=\eta \bar{s}_{l}^{i} \gamma_{\mu} u_{L}^{i} \bar{u}_{L}^{j} \gamma_{\mu} b_{L}^{j} \\
\eta=4 U_{u b} U_{u s}^{*} G_{F} / \sqrt{2}
\end{gathered}
$$

The effects of the tree level interaction could be large in general. We find however that there are certain modes of $B$ decays where penguin clearly dominate. In others tree is comparable or quite large. We evaluate the rates arising from these contributions independently and present our results in Table 1 . We shall define $U_{u b} / U_{b c}=0.1 \xi$, where $-1<\xi<1$. The modes where we find that penguin contribution clearly dominates are:

$$
\begin{array}{rlrl}
B^{+} \rightarrow & K^{0} \pi^{+}, K^{*+} \rho^{0} & B^{0} \rightarrow & \phi\left(K^{0}, K^{*+}\right) \\
& K^{* 0}\left(\pi^{+}, \rho^{+}\right) \\
& \phi\left(K^{0}, K^{*+}\right)
\end{array}
$$


If $U_{u b}$ turns out to be very small and penguin dominates all the two body decays, then we would have $\Delta I=0$ rule. We find, assuming $\Delta I=0$, the following isospin relations between the decay modes:

$$
\begin{aligned}
\Gamma\left(B^{+} \rightarrow K^{0} \pi^{+}\right) & =\Gamma\left(B^{0} \rightarrow K^{+} \pi^{-}\right)=2 \Gamma\left(B^{+} \rightarrow K^{+} \pi^{0}\right)=2 \Gamma\left(B^{0} \rightarrow K^{0} \pi^{0}\right) \\
\Gamma\left(B^{+} \rightarrow K^{+} \phi\right) & =\Gamma\left(B^{0} \rightarrow K^{0} \phi\right) \\
\Gamma\left(B^{+} \rightarrow K^{0} \rho^{+}\right) & =\Gamma\left(B^{0} \rightarrow K^{+} \rho^{-}\right)=2 \Gamma\left(B^{+} \rightarrow K^{+} \rho^{0}\right)=2 \Gamma\left(B^{0} \rightarrow K^{0} \rho^{0}\right) \\
\Gamma\left(B^{+} \rightarrow K^{* 0} \pi^{+}\right) & =\Gamma\left(B^{0} \rightarrow K^{*+} \pi^{-}\right)=2 \Gamma\left(B^{+} \rightarrow K^{*+} \pi^{0}\right)=2 \Gamma\left(B^{0} \rightarrow K^{* 0} \pi^{0}\right) \\
\Gamma\left(B^{+} \rightarrow K^{*+} \phi\right) & =\Gamma\left(B^{0} \rightarrow K^{* 0} \phi\right) \\
\Gamma\left(B^{+} \rightarrow K^{* 0} \rho^{+}\right) & =\Gamma\left(B^{0} \rightarrow K^{*+} \rho^{-}\right)=2 \Gamma\left(B^{+} \rightarrow K^{*+} \rho^{0}\right)=2 \Gamma\left(B^{0} \rightarrow K^{* 0} \rho^{0}\right)
\end{aligned}
$$

Departure from these relations will clearly show the importance of doubly suppressed Cabbibo transitions.

We have considered a large number of two body charmless decay modes of the $\mathrm{B}$ meson. Although in principle they can receive contributions from the penguin or the tree diagram, we found a number of modes where one of the contribution clearly dominates. Many of the branching ratios are in the interesting range of a few times $10^{-5}$, which should be accessible very soon, as seen from the present experimental limits. We further find a few modes that are highly suppressed, receiving negligible contribution from both the Hamiltonians. Lack of observation of these would test our method of computation. When the contributions are comparable, one of course should add the amplitudes and square. We have not done so because we do not know the magnitude and sign of $U_{u b}$, and there might be different final state phases on these amplitudes. A clean test is provided by those decays where one of the Hamiltonian dominates. In the case where the tree Hamiltonian dominates, one could even estimate $U_{b u}$. As our knowledge of these decays improves, we believe more ambitious methods might be used to calculate the amplitudes.

\section{Theory of CP Violation in $B^{ \pm}$Decays}

The basic theory follows from the work of Bander, Silverman and Soni[29]. The three conditions for asymmetry to arise are:

1. Two amplitudes with different Kobayashi-Maskawa phases must contribute to the same process.

2. There should be a complex phase in the Kobayashi-Maskawa matrix.

3. At least one of the amplitudes must have an absorptive part. (This is some times referred to as final state interaction, though I find this terminology misleading) 
Table 1: Branching ratios for charless two-body decay modes of the $B$ meson. Decay rates from the penguin and tree Hamiltonians are contributed separately. Some of the zeros stand for extremely small rates.

\begin{tabular}{cccc}
\hline $\begin{array}{c}\text { Branching } \\
\text { ratio } \\
\text { mode }\end{array}$ & $\begin{array}{c}\text { Contribution } \\
\text { from } H_{\text {eff }}^{T} \\
\left(10^{-5}\right)\end{array}$ & $\begin{array}{c}\text { Contribution } \\
\text { from } H_{\text {eff }}^{P} \\
\left(10^{-5}\right)\end{array}$ & $\begin{array}{c}\text { Present } \\
\text { experimental limit at } \\
90 \% \text { C.L. }\left(10^{-5}\right)\end{array}$ \\
\hline$B^{+} \rightarrow K^{+} \pi^{0}$ & $0.06 \xi^{2}$ & 0.53 & \\
$K^{0} \pi^{+}$ & 0 & 1.06 & 9.0 \\
$K^{+} \phi$ & 0 & 1.12 & 8.0 \\
$K^{+} \rho^{0}$ & $0.01 \xi^{2}$ & 0 & 7.0 \\
$K^{0} \rho^{+}$ & 0 & 0 & \\
$K^{*+} \pi^{0}$ & $0.05 \xi^{2}$ & 0.29 & \\
$K^{* 0} \pi^{+}$ & 0 & 0.58 & 13.0 \\
$K^{*+} \phi$ & 0 & 3.12 & \\
$K^{*+} \rho^{0}$ & 0 & 0.62 & \\
$K^{* 0} \rho^{+}$ & 0 & 1.24 & \\
& & & \\
$B^{0} \rightarrow \pi^{0}$ & 0 & 0.53 & \\
$K^{+} \pi^{-}$ & $0.06 \xi^{2}$ & 1.06 & \\
$K^{0} \phi$ & 0 & 1.12 & \\
$K^{0} \rho^{0}$ & $0.01 \xi^{2}$ & 0 & \\
$K^{+} \rho^{-}$ & 0 & 0 & \\
$K^{* 0} \pi^{-}$ & 0 & 0.29 & \\
$K^{*+} \pi^{-}$ & $0.10 \xi^{2}$ & 0.58 & \\
$K^{* 0} \phi$ & 0 & 3.12 & \\
$K^{* 0} \rho^{0}$ & 0 & 0.62 & \\
$K^{*+} \rho^{-}$ & $0.01 \xi^{2}$ & 1.24 & \\
\hline
\end{tabular}




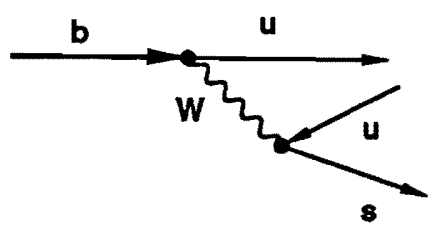

a) Tree Diagram

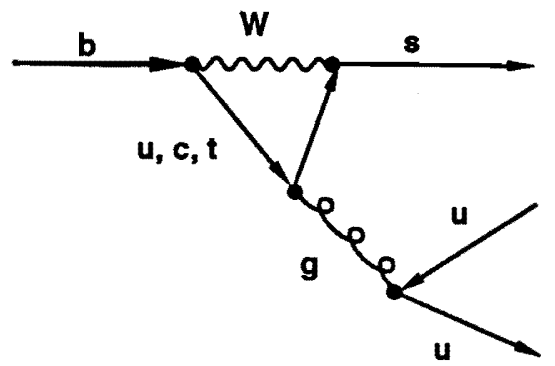

b) Penguin Diagram

Figure 4: Diagrams for $b \rightarrow s u \bar{u}$

The easiest way to generate an absorptive part, at least at the quark level is to consider one loop diagrams involving a gluon or a photon, usually referred to as penguin diagrams. The loop diagram generates an absorptive part when the virtual gluon four-momentum $q^{2}$ is greater than $4 m_{q}^{2}$ where $m_{q}$ is mass of the intermediate quark. For b quark decay, the intermediate quarks are u,c,t, so that $q^{2}>4 m_{u}^{2}$ or $4 m_{c}^{2}$ generates the absorptive part. The two amplitudes necessary for the effect can be a tree diagram and a penguin diagram, or if the tree diagram is absent, two penguin diagrams with different quark intermediate states suffice. The asymmetries are easiest to calculate at the quark level, but since the measurements will be made with a definite hadronic mode, the question of the reliability of such a calculation has to answered. We shall comment on this point in the conclusions.

Let us review the calculation with $b \rightarrow u \bar{u} s$ as a definite quark channel for illustrative purposes. The following diagrams contribute to this process:

We can write the amplitude for this process as

$$
M(b \rightarrow u \bar{u} s)=V_{u} A_{u}+\left(\alpha_{s} / \pi\right) \sum_{i} V_{i} P_{i}
$$

where $i$ runs over $u, c, t$ and $V_{i}$ is defined in terms of $K-M$ angles as

$$
V_{i}=U_{b i} U_{s i}^{*}
$$

Unitarity requires that

$$
\sum_{i} V_{i}=0
$$

$A_{u}$ refers to the contribution from the tree diagram, while $P_{i}$ refers to the contribution from the penguin diagram. We have factored $\left(\alpha_{s} / \pi\right)$ so that the remaining penguin operator contributes roughly the same as the tree operator. Using unitarity we can remove $V_{t}$ dependence, and we have

$$
M=V_{u}\left(A_{u}+\left(\alpha_{s} / \pi\right) P_{u-t}\right)+\left(\alpha_{s} / \pi\right) V_{c} P_{c-t}
$$


We now define $\bar{M}$ as the amplitude for $\bar{b} \rightarrow \bar{u} u \bar{s}$. This is given by

$$
\bar{M}=V_{u}^{*}\left(A_{u}+\left(\alpha_{s} / \pi\right) P_{u-t}\right)+\left(\alpha_{s} / \pi\right) V_{c}^{*} P_{c-t}
$$

The asymmetry is then given by

$$
A=\frac{|M|^{2}-|\bar{M}|^{2}}{|M|^{2}+|\bar{M}|^{2}}
$$

In the denominator we may safely assume that $\left(\alpha_{s} / \pi\right) V_{c} \gg V_{u}$, and obtain

$$
A=\frac{2 \operatorname{Im}\left(V_{u} V_{c}^{*}\right) \operatorname{Im}\left[\left(A_{u}^{*}+\left(\alpha_{s} / \pi\right) P_{u-t}^{*}\right)\left(\alpha_{s} / \pi\right) P_{c-t}\right]}{\left|V_{c}\right|^{2}\left(\alpha_{s} / \pi\right)^{2}\left|P_{c-t}\right|^{2}}
$$

This expression is clearly zero unless the three conditions we mentioned above are satisfied. The maximum asymmetry occurs when tree amplitude is present and $P_{c-t}$ has absorptive part, which requires $q^{2}>4 m_{c}^{2}$. If $q^{2}<4 m_{c}^{2}$, the formula generates absorptive part due to $\left(\alpha_{s} / \pi\right)^{2}$ contribution, however, to this order there are other contributions which must be evaluated [30]. In pure penguin process, like $b \rightarrow s \bar{s} s$, we have the expression

$$
A=\frac{2 \operatorname{Im}\left[V_{u} V_{c}^{*}\right] \operatorname{Im}\left[P_{u-t}^{*} P_{c-t}\right]}{\left|V_{c}\right|^{2} P_{c-t}^{2}}
$$

The asymmetry is smaller, but the estimates are less model dependent in this case.

\subsection{Pure Penguin Interference}

These processes are much cleaner because although the rate for a given mode is dependent on hadronic wave functions, the asymmetries are not. Let us first consider the decay $b \rightarrow s \phi$ which arises at the quark level from $b \rightarrow s \bar{s} s$. We can isolate this quark process by looking at $B \rightarrow K \phi$ or $K^{*} \phi$. The quark level lagrangian is given by [31].

$$
L=\kappa I\left(k^{2}\right) \bar{s} \lambda^{a} \gamma_{\mu}\left(1-\gamma_{5}\right) b \bar{s} \lambda^{a} \gamma^{\mu} s
$$

where $\kappa=\left(G_{F} / \sqrt{2}\right)\left(\alpha_{s} / 8 \pi\right)$ and

$$
\begin{gathered}
I\left(k^{2}\right)=\sum_{i} V_{i} F_{i}\left(k^{2}\right) \\
F_{i}\left(k^{2}\right)=-\int_{0}^{1} d y \int_{0}^{1-y} d x \frac{\left[2 y(x-1)+4 x(1-x)+\widehat{m}_{i}^{2}(x y+2 x(x-1))\right]}{y+\hat{k}^{2} x(x-1)+\widehat{m}_{i}^{2}(1-y)}
\end{gathered}
$$




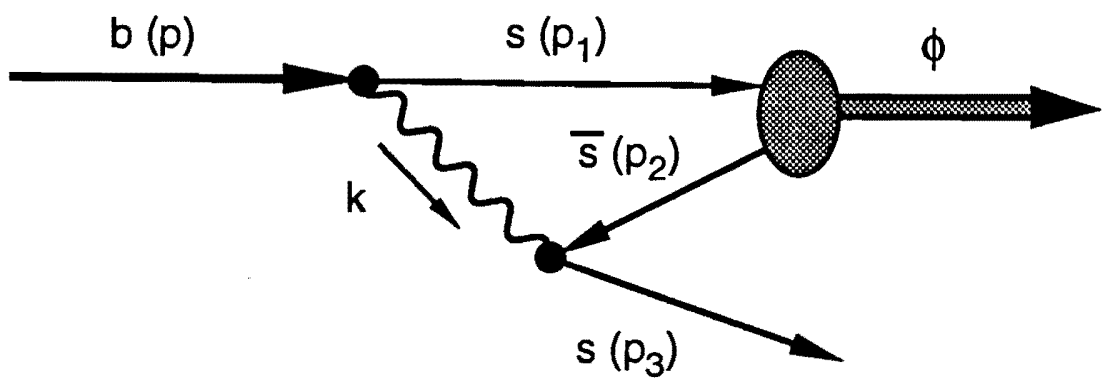

Figure 5: The process $b \rightarrow s \phi$

where $\hat{k}^{2}=k^{2} / M_{W}^{2}, \widehat{m}_{i}^{2}=m_{i}^{2} / M_{W}^{2}$, and $\mathrm{i}=\mathrm{u}, \mathrm{c}$,t. The asymmetry for the process $b \rightarrow s \phi$ can be calculated provided we know what value of $k^{2}$ to use. We use Fig. 5 to illustrate the process and argue from the fact that $\phi$ can be only formed from $s$ quark coming from $\mathrm{b}$ and $\bar{s}$ coming from the gluon, since $\phi$ is in a color singlet state. Simple kinematics yields $k^{2}=\left(m_{b}^{2}-m_{s}^{2}\right) / 2 \approx 12(G e v)^{2}$. We can now evaluate the dispersive and the absorptive part of the penguin diagrams. For $\widehat{m}_{i} \ll 1$, we have

$$
\begin{gathered}
\operatorname{Re} F_{1}\left(k^{2}\right)=-4 \int_{0}^{1} d x x(1-x) \ln \left|\widehat{m}_{i}^{2}-\hat{k}^{2} x(1-x)\right| \\
\operatorname{Im} F_{1}\left(k^{2}\right)=-(\pi / 2) \theta\left(k^{2}-4 M_{i}^{2}\right)\left(b_{+}^{2}-b_{-}^{2}+b_{-}^{3} / 3-b_{+}^{3} / 3\right)
\end{gathered}
$$

where $b_{ \pm}=1 \pm\left(1-4 m^{2} / k^{2}\right)^{1 / 2}$. We now find:

\begin{tabular}{|c|c|}
\hline Quantity & Value \\
\hline $\operatorname{Re} F_{1}^{u}$ & 5.3 \\
$\operatorname{Im} F_{1}^{u}$ & -2.1 \\
$\operatorname{Re} F_{1}^{c}$ & 6.4 \\
$\operatorname{Im} F_{1}^{c}$ & -1.4 \\
$F_{1}^{t}$ & .71 \\
\hline
\end{tabular}

where we have chosen $m_{t} \approx M_{W}$. We find for the asymmetry

$$
A=\frac{2 \operatorname{Im}\left[V_{u} V_{c}^{*}\right](5 / 33)}{\left|V_{c}\right|^{2}}
$$

If we use Wolfenstein parametrization,

$$
U=\left(\begin{array}{ccc}
1 & \lambda & A \lambda^{3}(\rho-i \eta) \\
-\lambda & 1 & A \lambda^{2} \\
A \lambda^{3}(1-\rho-i \eta) & -A \lambda^{2} & 1
\end{array}\right)
$$

then

$$
A=\lambda^{2} \eta(5 / 33)=\eta \times 10^{-2}
$$


the value of $\epsilon, B-\bar{B}$ mixing, $m_{t}$ and $U_{b u}$ imply $A=1.0 \pm 0.1$ and $\eta>0.1$ The asymmetry is therefore $\geq 0.1 \%$. If we had calculated the asymmetry of the Cabibbo suppressed penguin $b \rightarrow d s \bar{s}$ process, the asymmetry would be of order $\eta$ [32]. However the branching ratio of any exclusive mode like $B \rightarrow K \bar{K}$ from this process is suppressed and is expected to be of the order of $10^{-7}$. The exclusive modes $B^{-} \rightarrow K^{-} \phi$ or $B^{-} \rightarrow K^{*-} \phi$ are expected to have a branching ratio of order $10^{-5}[31]$ and the asymmetry equal to the inclusive process $b \rightarrow s \phi$.

\subsection{Processes With Tree-Penguin Interference}

The largest observable asymmetry is expected to arise in processes where tree diagram and penguin contribute approximately the same amount. At the quark level the process is $b \rightarrow s \bar{u} u$, and the asymmetry is given by (33), which can be written as

$$
A=\eta\left(A_{u} /\left|P_{c-t}\right|\right)\left(P_{c-t}^{A} /\left|P_{c-t}\right|\right)
$$

We need the ratio of tree and penguin contributions, as well as the absorptive to dispersive ratio. The former can be calculated in from the lagrangian if factorization approximation is used. A large number of processes have been evaluated in [28] using this approximation. We present below the best modes with the corresponding ratio $R=A_{u} /\left|P_{c-t}\right|$. To calculate the absorptive part we need to know the value of $q^{2}$ to be used. If the argument used in the previous section can be applied, $12(\mathrm{GeV})^{2}$ might be suitable. The value of $P_{c-t}^{A} /\left|P_{c-t}\right| \approx 0.25$ in this case.

\begin{tabular}{|c|c|}
\hline \multicolumn{1}{|c|}{ Mode } & $R=A_{u} /\left|P_{c-t}\right|$ \\
\hline$B^{-} \rightarrow K^{-} \pi^{\circ}$ & 1.1 \\
$B^{-} \rightarrow K^{*-} \pi^{\circ}$ & 1.4 \\
$B^{\circ} \rightarrow K^{+} \pi^{-}$ & 0.8 \\
$B^{\circ} \rightarrow K^{*+} \pi^{-}$ & 1.4 \\
\hline
\end{tabular}

The asymmetry then is given by

$$
A \cong \eta R / 4
$$

This could be as high as $3.5 \%$ for $\eta=0.1$, and clearly in an observable range.

The above estimate are based on the applicability of the one loop quark diagram to a hadronic decay, a procedure that has been criticized recently by Wolfenstein [33]. In my opinion the use of one loop diagram is clearly possible only if the quark diagram is a short distance operator and long distance effects are negligible. This might be true for a heavy quark decay where large momenta are involved in the decay products. A more troubling question of the appropriate $k^{2}$ to be used will have to deferred until a better understanding emerges regarding hadronic decays. Importance of detecting direct CP violation outweighs the fact that the theoretical 
calculations are not at present very reliable. We expect the situation to improve as the penguin processes are measured.

Research contributing to this article has been supported by a grant from the Department of Energy.

\section{References}

[1] C. Dib, I. Dunitz and F. Gilman, Phys. Rev. D39, 2639 (1989)

[2] M. Bauer, B. Stech and M. Wirbel, Z. Phys. C29, 637 (1985); ibidC34 , 103 (1987).

[3] B. Grinstein, M.B. Wise and N. Isgur, Phys. Rev. Lett.56, 298 (1986) and California Institute of Technology Report No. CALT-68-1311, 1985.

[4] M.B. Voloshin and M.A. Shifman, Yad. Fiz.45, 463 (1987); N. Isgur and M.B. Wise, Phys. Lett.B232, 113 (1989);B237, 537 (1990).

[5] R. Ammar et. al., Phys. Rev. Lett71, 674 (1993).

[6] T. Inami and C.S. Lim, Prog. Theor. Phys. 65, 297 (1981); N.G. Deshpande and G. Eilam, Phys. Rev.D26, 2463 (1982); N.G. Deshpande and M. Nazerimonfared, Nucl. Phys.B123, 390 (1983).

[7] N.G. Deshpande, P. Lo, J. Trampetic, G. Eilam and P. Singer, Phys. Rev. Lett.59, 193 (1987).

[8] S. Bertolini, F. Borzumati and A. Masiero, Phys. Rev. Lett.59, 180 (1987).

[9] M.A. Shifman, A.I. Vainshtein and V.I. Zakharov, Phys. Rev.D18, 2583 (1978).

[10] B. Grinstein, R. Springer and M.B. Wise, Phys. Lett.202B, 138 (1988) and Nuclear PhysicsB339, 269(1990); M. Misiak, Nuclear PhysicsB393, 23(1993).

[11] R. Grigjanis, P.J. O'Donnell, M. Sutherland and H. Navalet, Phys. Lett.B213, 355 (1988) and Phys. Lett.B 237, 252 (1990).

[12] A.J. Buras, M. Misiak, M. Muntz and S. Pokorski, Max-Plank Institute preprint, MPI-Ph/93-77

[13] N.G. Deshpande, P. Lo and J. Trampetic, Z. Phys.C40, 369 (1988).See also Brief Review by N. G. Deshpandeand J. Trampetic in Mod. Phys. Lett.A4, 2095 (1989). For a discussion of long distance contributions see N.G. Deshpande, K. Panose and J. Trampetic, Phys. Lett. B214, 467 (1988) 
[14] C.A. Dominquez, N. Paver and Riazuddin, Phys. Lett,B214, 459 (1988) T. Altomari , Phys.Rev.D37,677(1988).

[15] R. Caslbuoni et.al.,preprint UGVA-DEPT 1993/04-816

[16] A. Ali, C. Greub and T. Mannel, DESY preprint, DESY-93-016.

[17] C. Bernard, P. Hsieh and A. Soni, Washington University preprint, HEP/9335

[18] N.G. Deshpande and J. Trampetic, Phys. Rev. D40,3773(1989).

[19] S. Bertolini, F. Borzumati and A. Masiero, Phys. Lett.192B, 69 (1987). M.A. Diaz, Vanderbilt University preprint, VAND-TH-93-11.

[20] J.L. Hewett; Phys. Rev. Lett.70, 1045 (1993); V. Barger, M.S. Berger and R.J.N. Phillips, Phys. Rev. Lett. 70, 1368 (1993).

[21] X-G. He and B. McKellar, University of Melbourne preprint, UM-P-93/52; T. Rizzo, Argonne National Lab. preprint, ANL-HEP-PR-93-19.

[22] B. Grinstein, M.J. Savage and M.B. Wise; Nucl. Phys.319B, 271 (1989).

[23] N.G. Deshpande, J. Trampetic, and K. Panose; Phys. Rev.D39, 1461 (1989).

[24] N.G. Deshpande and J. Trampetic; Phys. Rev. Lett. 60, 2583 (1988). See also R. Grigjanis et. al., Phys. Rev. D41, 245 (1990); W. Jaus and D.Wyler, Phys. Rev.D41,3405 (1990). and N.G. Deshpande, K. Panose and J. Trampetic, Phys. Lett.B308, 322 (1993)

[25] C. Albajar et. al., Phys. Letts.262B, 163 (1991).

[26] B. Guberina,R. Peccei and R. Ruckl, Phys. Lett. B90,169(1980);G. Eilam, Phys. Rev. Lett.49,1478(1982)

[27] W.S. Hou, Nucl. Phys.B308, 561 (1988).

[28] N.G. Deshpande and J. Trampetic, Phys. Rev.D41, 895(1990).

[29] M. Bander, D. Silverman, and A. Soni, Phys. Rev. Lett.43, 142 (1979).

[30] J.M. Gerard and W.S. Hou, Phys. Rev. Lett.,62, 855 (1989).

[31] N.G. Deshpande and J. Trampetic, Phys. Rev.D41, 2926 (1990).

[32] J.M. Gerard and W.S. Hou, Phys. Lett. B253, 478(1991);Phys. Rev.D43, 2909 (1991).

[33] L. Wolfenstein, preprint, NSF-ITP-90-29 